\title{
Dependence Levels as Interim Clinical Milestones Along the Continuum of Alzheimer's Disease: 18-Month Results from the GERAS Observational Study
}

\author{
K. Kahle-Wrobleski', J.S. Andrews ${ }^{1}$, M. Belger' ${ }^{2}$,W. Ye ${ }^{1}$, S. Gauthier ${ }^{3}$, D.M. Rentz ${ }^{4}$, D. Galasko ${ }^{5}$

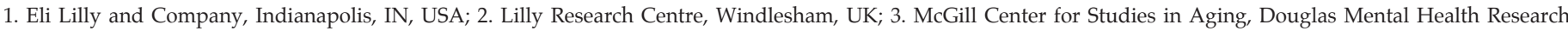 \\ Institute, Montreal, QC, Canada; 4. Harvard Medical School, Boston, MA, USA; 5. University of California San Diego, San Diego, CA, USA

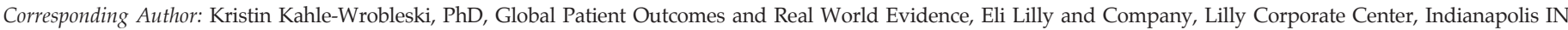 \\ 46285, USA, Phone: 317-651-9881, Fax: 317-276-5791, Email: wrobleski_kristin_kahle@lilly.com \\ J Prev Alz Dis 2017;4(2):72-80 \\ Published online January 24, 2017, http:/ / dx.doi.org/10.14283/jpad.2017.2
}

\begin{abstract}
BACKGROUND: While functional loss forms part of the current diagnostic criteria used to identify dementia due to Alzheimer's disease, the gradual and progressive nature of the disease makes it difficult to recognize clinically relevant signposts that could be helpful in making treatment and management decisions. Having previously observed a significant relationship between stages of functional dependence (the level of assistance patients require consequent to Alzheimer's disease deficits, derived from the Alzheimer's Disease Cooperative Study Activities of Daily Living Scale) and cognitive severity, we investigated whether measures of functional dependence could be utilized to identify clinical milestones of Alzheimer's disease progression.

OBJECTIVES: To describe the patterns of change in dependence over the course of 18 months in groups stratified according to cognitive Alzheimer's disease dementia severity (determined using the Mini-Mental State Examination score) and to identify characteristics associated with patients showing worsening dependence (progressors) versus those showing no change or improvement (non-progressors).
\end{abstract}

DESIGN: Analysis of longitudinal data from the GERAS study.

SETTING: GERAS is an 18-month prospective, multicenter, naturalistic, observational cohort study reflecting the routine care of patients with Alzheimer's disease in France, Germany, and the United Kingdom.

PARTICIPANTS: 1495 community-living patients, aged $\geq 55$ years, diagnosed with probable Alzheimer's disease dementia, and their caregivers.

MEASUREMENTS: Dependence levels, cognitive function, behavioral symptoms, caregiver burden, and cost were assessed at baseline and at 18 months.

RESULTS: Of 971 patients having both baseline and 18-month data, 42\% (408) were progressors and 563 (58\%) were nonprogressors. This general pattern held for all three levels of baseline Alzheimer's disease dementia severity - mild (MiniMental State Examination score 21-26), moderate (15-20) or moderately severe/severe $(<15)$ - with $40-45 \%$ of each group identified as progressors and $55-60 \%$ as non-progressors. No baseline differences were seen between progressors and non-progressors in cognitive scores or behavioral symptoms, although progressors had significantly shorter times since diagnosis and showed milder functional impairment. Baseline factors predictive of increasing dependence over 18 months included more severe cognitive impairment, living with others, and having multiple caregivers. A higher level of Received November 22, 2016

Accepted for publication December 1, 2016 initial dependence was associated with less risk of dependence progression. Total societal costs of care also increased with greater dependence.

CONCLUSIONS: In this large cohort, $42 \%$ of Alzheimer's disease dementia patients at all levels of cognitive severity became more dependent within 18 months of observation while $58 \%$ did not progress. Dependence levels may be considered as meaningful interim clinical milestones that reflect Alzheimer's disease-related functional deficits, although a time frame that extends beyond 18 months may be necessary to observe changes if used in clinical trials or other longitudinal studies. Recognition of predictors of greater dependence offers opportunities for intervention.

Key words: Alzheimer disease, dependence, observational study, ADCS-ADL, GERAS.

\section{Introduction}

7 The core clinical criteria for making a diagnosis of dementia due to Alzheimer's disease (AD) include declines in memory and other cognitive abilities, impairments in activities of daily living (ADL) and global functioning, as well as uncharacteristic changes in behavior or personality (1). As AD is a progressive and chronic illness, it is challenging to characterize clinically significant and discrete milestones of disease progression $(2,3)$. Nevertheless, defining some clinically relevant $\mathrm{AD}$ milestones may be useful to physicians, caregivers, and healthcare systems, as well as patients themselves, in making appropriate and timely treatment and management decisions.

Functional loss forms part of the current diagnostic criteria used to identify dementia due to AD (1); however, it is defined with less granularity than cognition, which includes standard domains such as memory and language (4). Yet, accurate measurement of functional loss can help determine the type, level, and costs associated with current and future care of a patient with $\mathrm{AD}$ dementia. Measures of meaningful clinical change 
in clinical trials of AD dementia have not been well established (5); hence, both the European Medicines Agency and the US Food and Drug Administration recommend that changes in cognition as well as function are needed for approval of a treatment for cognitive symptoms in $\mathrm{AD}$ dementia.

A variety of scales, developed to assess function in patients with $\mathrm{AD}$ dementia, have been incorporated into clinical trials and observational studies seeking to evaluate the impact of treatments or interventions. They include the Functional Activities Questionnaire; Disability Assessment for Dementia scale; Lawton scale; Global Deterioration Scale/Functional Assessment Staging system; Clinical Dementia Rating scale; Blessed Dementia Rating Scale; Amsterdam IADL (Instrumental Activities of Daily Living) Questionnaire; and the Alzheimer's Disease Cooperative Study - Activities of Daily Living Scale (ADCS-ADL).

Assessing dependence, which refers to the level of assistance a patient requires due to AD dementiarelated deficits, is an alternative, pragmatic approach to monitoring $\mathrm{AD}$ progression. As patients become more dependent, they face increased need for home assistance or possible institutionalization (or equivalent institutional care), along with higher healthcare, informalcare, and total-care costs (6-9). Increased dependence is accompanied by changes in family dynamics, and raises ethical and legal issues regarding financial and healthcare decision-making and guardianship (10). Dependence is thought to be influenced by - but different from - cognition, functioning, or behavior (6, 11, 12). It has been described as a distinct measurable component of dementia and an important determinant of AD-related disability (13). In the DADE Study, Jones et al. (8) observed significant associations between dependence, assessed by the Dependence Scale (DS) (13), and service use cost, patient quality of life (QoL), and caregiver perceived burden. They also suggested that, as a construct, dependence could be used to reflect the combined effects of cognitive functional, and behavioral changes seen in AD dementia into a more easily interpretable form.

Our previous work, a cross-sectional analysis of baseline data from the GERAS study (14), showed that dependence levels can be adapted from functional scales (15). Our results showed a significant relationship between assigned levels of dependence, derived from the ADCS-ADL score, and cognitive severity category in a large cohort of $\mathrm{AD}$ dementia patients receiving routine care. Importantly, with a greater assigned level of dependence, clinical and economic indicators showed a pattern of greater disease severity and higher costs. This work provided initial support for the use of dependence levels as appropriate interim clinical milestones that characterize the functional deficits associated with $\mathrm{AD}$ dementia. Our goal is to develop a system that can be used to stage disease progression in patients with $\mathrm{AD}$ dementia that is informative to both care providers and stakeholders, and that can also be used as a common metric in clinical trials or other research studies.

This report from the current longitudinal phase of the GERAS study expands the above-mentioned analysis with data from 18 months of evaluation. The objectives were to describe patterns of change in dependence within an 18-month period in groups stratified according to baseline cognitive AD dementia severity, to examine the correlation between dependence level and other outcome measures, and to identify characteristics that differentiated patients with $\mathrm{AD}$ dementia who showed worsening dependence (progressors) from those who showed no change or improved (non-progressors).

\section{Methods}

\section{GERAS Study Design and Participants}

The GERAS study was an 18-month, prospective, multicenter, naturalistic, observational, cohort study, which reflected the routine care of patients with $A D$ dementia in France, Germany, and the United Kingdom (UK). The study was extended by a further 18-month follow-up period in France and Germany. The study design, methods, and baseline patient characteristics were previously described $(14,15)$. Patients were enrolled from October 2010 until September 2011. Patients and their caregivers were evaluated at baseline and during three care visits at 6-month intervals as part of their routine care. The study centers involved were mostly specialist secondary care clinics ("memory clinics").

Briefly, patients were community-dwellers aged $\geq 55$ years with probable AD dementia (National Institute of Neurological and Communicative Disorders and Stroke and Alzheimer's Disease and Related Disorders Association [NINCDS-ADRDA] criteria) (16), a MiniMental State Examination (MMSE) score of $\leq 26$ (17), and who presented within the normal course of care. Those with other potential causes of dementia were excluded from the study.

Each patient was also required to have a primary caregiver who agreed to participate in the study and to undertake responsibility for the patient for at least 6 months of the year. All patients (or their legal representatives) and caregivers gave written informed consent before entering the study, which was approved by ethical review boards in each country according to country-specific regulations.

\section{Patient and Caregiver Data}

Patients were stratified according to AD dementia severity, based in part on National Institute for Health and Care Excellence (NICE) guidance 217 (18), as having mild (MMSE score 21-26), moderate (15-20) 
or moderately severe/severe $(<15)$ AD dementia. The study aimed to achieve approximately equal numbers of patients in the three AD dementia severity groups within each country. Functional ability was assessed using the caregiver-rated ADCS-ADL (19), with basic (BADL) and instrumental (complex) (IADL) sub-scales. Cognitive function was evaluated using the MMSE (17) in patients with mild or moderate $\mathrm{AD}$ dementia. The proxy version of the EuroQol-5 Dimension (EQ-5D) was used to obtain information from caregivers regarding patients' health status (20). Behavioral symptoms were evaluated using the 12-item Neuropsychiatric Inventory (NPI-12) (21). Direct and indirect patient and caregiver resource use was measured using the Research Utilization in Dementia Scale (RUD) (22). To evaluate the impact on caregivers, total caregiver time and caregiver supervision time from the RUD were measured, as well as caregiver burden using the Zarit Burden Interview (ZBI) (23). Data were generally collected at baseline, 6, 12 and 18 months, although ADCS-ADL and EQ-5D data were measured only at baseline and 18 months.

\section{Cost Estimations}

For each country, monthly cost values were estimated by applying unit costs of services and products (2010 values) to the health and social care resource use data collected over the 18-month follow-up period. For resource use items, full details of the unit costs applied and their sources for each country have already been reported (14). All UK costs in pounds sterling $(£)$ were converted to Euros $(€)$ using the conversion rate $£ 1=€ 1.1661$, calculated as the average monthly exchange rate for 2010 .

In this analysis, we looked at direct medical costs and total societal costs. Total societal costs were calculated by adding patient healthcare costs (including medications, hospitalizations, and outpatient visits), patient social care costs (including community care services, structural adaptations to the home, and extra financial support), and caregiver informal care costs (excluding caregiver direct healthcare costs).

Caregiver time, calculated as the number of hours for BADL and IADL, was capped at 24 hours/day; supervision time was excluded from cost calculations. The unit costs of caregiver time for working caregivers was the value of lost production time based on the national average wage per country population; for nonworking caregivers, it was the value of lost leisure time based on $35 \%$ of the national average wage per country population.

The following imputation rules were applied for missing data: for institutionalized patients, mean monthly costs from the last visit were used for the period until institutionalization, then monthly costs of institutionalization were used from institutionalization up to 18 months; for patients who died, last observation carried forward (LOCF) was used such that costs from the last known visit were extrapolated up to the date of death (no costs after death were computed); for patients lost to follow-up, multiple imputation stratified by MMSE group and country was performed on missing costs. The factors used in the multiple imputation procedure were selected from those recently identified by Dodel et al. (24).

\section{Categorizing Dependence Levels}

Exploratory factor analysis of ADCS-ADL data was conducted in order to create subscales to aid the construction of dependence levels (4), details of which were described in our earlier analysis (15). In brief, baseline data suggested a 4-factor solution that included subdomains for specific competencies, including BADLs (eating, walking, toileting, bathing, grooming and dressing), domestic/household activities (choosing clothes, using the telephone, clearing the dishes, finding belongings, cooking, putting out the rubbish, using appliances), communication/engagement with the environment (watching television, paying attention to conversation, keeping appointments, talking about current events, reading, writing, performing hobbies), and outdoor activities (going out, shopping, paying, being left alone) (15).

The DS includes a scheme to derive levels of dependence based on the ability to perform ADLs (13), such as those represented within the ADCS-ADL (19). In an earlier study, we described six theory-driven assigned levels of dependence beginning with Level 0 (no care needed and completely independent), with dependence increasing incrementally over levels 1 through 5, which represents complete dependence, such as the needs of someone living in a nursing home (15). In the present analysis, the level of functional dependence, calculated from the ADCS-ADL, was determined for each patient at baseline and at 18 months.

A functional progressor was defined as someone whose change in functional dependence from baseline to the 18-month follow-up increased by 1 or more levels, while a non-progressor showed either no change or a decrease in 1 or more dependence levels.

\section{Statistical Analysis}

All patients and associated caregivers who provided informed consent and fulfilled the study entry criteria were included in the present analysis. All calculations were based on non-missing observations. Since dependence levels were derived from the ADCS-ADL, this analysis was limited to patients with ADCS-ADL data collected at baseline and at 18 months. Descriptive statistics (mean and standard deviation [SD] or frequency) for two groups (those for whom dependence level improved or did not change [non-progressors] 
Table 1. Mean baseline demographics (SD) for dependence non-progressors and progressors

\begin{tabular}{|c|c|c|c|}
\hline & \begin{tabular}{|l}
$\begin{array}{l}\text { Non- } \\
\text { progressors }\end{array}$ \\
\end{tabular} & Progressors & p-Value \\
\hline Patients, $\mathrm{n}$ & 563 & 408 & \\
\hline Age, years & $77.11(7.69)$ & $76.49(7.55)$ & 0.2164 \\
\hline Gender, female & $55.24 \%$ & $53.19 \%$ & 0.5262 \\
\hline Time since diagnosis, years & $2.38(2.39)$ & $2.02(2.00)$ & 0.0096 \\
\hline MMSE & $18.46(5.97)$ & $18.14(5.74)$ & 0.4032 \\
\hline BADL & $17.25(5.26)$ & $18.92(4.07)$ & $<0.0001$ \\
\hline IADL & $29.81(15.04)$ & $32.53(14.30)$ & 0.0046 \\
\hline Total ADL & $47.12(19.45)$ & $51.52(17.53)$ & 0.0002 \\
\hline Patient education & $10.41(3.24)$ & $10.60(3.04)$ & 0.3583 \\
\hline Number of comorbidities & $1.51(1.22)$ & $1.31(1.18)$ & 0.0117 \\
\hline \multicolumn{4}{|l|}{ NPI } \\
\hline NPI-12 total score & $12.81(12.64)$ & $12.67(12.22)$ & 0.8663 \\
\hline NPI psychosis score & $1.76(3.55)$ & $1.82(3.76)$ & 0.8087 \\
\hline NPI affective score & $2.64(3.67)$ & $2.67(3.66)$ & 0.8989 \\
\hline NPI apathy score & $4.50(4.94)$ & $4.15(4.48)$ & 0.2568 \\
\hline NPI hyperactivity score & $3.92(5.57)$ & $4.01(5.77)$ & 0.8187 \\
\hline Dependence level & & & $<0.0001$ \\
\hline 0 & $0.00 \%$ & $1.72 \%$ & \\
\hline 1 & $0.18 \%$ & $6.13 \%$ & \\
\hline 2 & $26.92 \%$ & $43.87 \%$ & \\
\hline 3 & $23.71 \%$ & $29.66 \%$ & \\
\hline 4 & $39.57 \%$ & $18.63 \%$ & \\
\hline 5 & $9.63 \%$ & $0.00 \%$ & \\
\hline AD dementia severity* & & & 0.3334 \\
\hline Mild & $44.76 \%$ & $40.44 \%$ & \\
\hline Moderate & $30.73 \%$ & $34.31 \%$ & \\
\hline Moderately severe/severe & $24.51 \%$ & $25.25 \%$ & \\
\hline Country & & & 0.7900 \\
\hline France & $29.31 \%$ & $27.70 \%$ & \\
\hline Germany & $35.17 \%$ & $37.01 \%$ & \\
\hline UK & $35.52 \%$ & $35.29 \%$ & \\
\hline Patient marital status & & & 0.0797 \\
\hline Divorced/separated & $2.13 \%$ & $2.45 \%$ & \\
\hline Married/cohabiting & $73.89 \%$ & $78.43 \%$ & \\
\hline Never married & $1.78 \%$ & $1.23 \%$ & \\
\hline Widowed & $22.20 \%$ & $17.89 \%$ & \\
\hline Patient lives alone & & & 0.0822 \\
\hline Yes & $16.52 \%$ & $12.50 \%$ & \\
\hline No & $83.48 \%$ & $87.50 \%$ & \\
\hline Any $\mathrm{AD}$ treatment & & & 0.0963 \\
\hline Yes & $86.15 \%$ & $89.71 \%$ & \\
\hline No & $13.85 \%$ & $10.29 \%$ & \\
\hline Patient has falls & & & 0.7904 \\
\hline Yes & $12.08 \%$ & $11.52 \%$ & \\
\hline No & $87.92 \%$ & $88.48 \%$ & \\
\hline \multicolumn{4}{|l|}{ Caregivers } \\
\hline Age, years & $67.55(12.02)$ & $68.40(11.25)$ & 0.2625 \\
\hline Gender, female & $64.59 \%$ & $62.01 \%$ & 0.4100 \\
\hline NPI caregiver distress score & $7.87(7.64)$ & $7.68(6.93)$ & 0.6891 \\
\hline Number of caregivers involved & & & 0.8752 \\
\hline 0 & $61.57 \%$ & $63.24 \%$ & \\
\hline
\end{tabular}

Table 1. Continued

\begin{tabular}{|c|l|l|l|}
\hline & $\begin{array}{l}\text { Non- } \\
\text { progressors }\end{array}$ & Progressors & p-Value \\
\hline 1 & $22.60 \%$ & $20.83 \%$ & \\
\hline 3 & $8.54 \%$ & $8.33 \%$ & \\
\hline 4 & $4.09 \%$ & $4.17 \%$ & \\
\hline $\begin{array}{c}\text { Caregiver works for pay } \\
\text { Yes }\end{array}$ & $3.20 \%$ & $3.43 \%$ & \\
\hline $\begin{array}{c}\text { No } \\
\text { Caregiver lives with patient }\end{array}$ & $21.89 \%$ & $21.57 \%$ & 0.9059 \\
\hline $\begin{array}{c}\text { Yes } \\
\text { No }\end{array}$ & $78.11 \%$ & $78.43 \%$ & \\
\hline $\begin{array}{c}\text { Caregiver relationship } \\
\text { Spouse }\end{array}$ & $77.94 \%$ & $80.64 \%$ & 0.3075 \\
\hline Child & $22.06 \%$ & $19.36 \%$ & \\
\hline Other & $69.09 \%$ & $73.53 \%$ & 0.1223 \\
\hline
\end{tabular}

Abbreviations: AD, Alzheimer's Disease; ADL, activities of daily living; BADL, basic ADL; IADL, instrumental ADL; MMSE, Mini-Mental State Examination; NPI, Neuropsychiatric Inventory; SD, standard deviation. *Alzheimer's Disease dementia severity based on MMSE scores: mild (21-26), moderate (15-20) and moderately severe/ severe $(<15)$.

and those for whom dependence level worsened [progressors]) were used to summarize baseline continuous variables (time since diagnosis, age, patient education, MMSE, ADL [total, basic, and instrumental] scores, and NPI total score and subscores) and categorical variables (gender, country, marital status, patient lives alone, any AD treatment, patient has falls, dependence level, and AD severity).

The distribution of patients at each level of dependence at baseline and 18 months for the overall patient sample and also according to cognitive severity at baseline were described. The percentage of patients with worsening dependence levels was also calculated.

Correlations between dependence levels and certain key outcome variables at baseline and at 18 months were examined using Pearson correlation coefficients. Generalized Linear Models (GLMs) with normal distribution and identity link function were used to examine whether changes in dependence level could predict changes in outcomes measures. The outcome measures were selected to reflect a range of outcomes that we expected would be related to level of dependence, including cognition (MMSE), caregiver burden (ZBI), overall caregiver time, NPI-12 total scores, patientreported QoL (EQ-5D), patient direct medical costs, and total societal costs. GLMs were controlled for the core list of patient baseline characteristics (outcome measures, country, age, gender, cognitive severity, number of comorbidities, and total ADCS-ADL scores) and caregiver baseline characteristics (age, caregiver spouse [yes/ no], and caregiver works for pay [yes/no]). In these models, changes in dependence levels and other outcome 
measures were considered as continuous variables to facilitate interpretation of the findings.

A stepwise logistic regression analysis was applied to the data to identify factors associated with patient progression versus non-progression. Baseline factors considered included: patient and caregiver age, gender, and country; patient cognitive MMSE severity (mild, moderate, moderately severe/severe), level of dependence, time since AD diagnosis, and NPI-12 scores (total and subdomains); indicator variables related to the patient (marital status, education, lives alone, comorbidities, receipt of AD medication, history of falling) and caregiver (lives with patient, is patient's spouse, works for pay); number of caregivers other than the primary caregiver; NPI-12 caregiver distress score; and ZBI scores. ADCS-ADL scores were not included as the baseline dependence levels included in the model were calculated using these scores.

All data were analyzed using SAS software, version 9.2 (SAS Institute, Cary, North Carolina, USA).

\section{Results}

Overall, 1532 patients and their primary caregivers were enrolled by 94 investigators. After excluding 35 patients who did not fulfill the study entry criteria and 2 patients following the baseline database lock, 1497 patients with probable AD dementia and caregivers (14) were included in the baseline analyses. Of these, 971 had both baseline and 18-month ADCS-ADL data. In the current analysis, 563/971 (57.98\%) patients showed no change $(479 / 971,49.33 \%)$ or improvement $(84 / 971$, $8.65 \%$ ) in dependence levels over 18 months (nonprogressors) while 408/971 (42.02\%) exhibited worsening of dependence levels (progressors).

\section{Descriptive Statistics}

A summary of baseline characteristics of patients and caregivers for progressors versus non-progressors is shown in Table 1. Full baseline statistics were previously reported $(14,15)$.

Non-progressors and progressors were similar in age, gender, years of education and marital status as well as caregiver characteristics. Most patients were married or lived with another person. Progressors had significantly shorter times since diagnosis than non-progressors $(\mathrm{p}=0.0096)$ and showed milder functional impairment at baseline, as indicated by total ADL ( $\mathrm{p}=0.0002)$, BADL $(\mathrm{p}<0.0001)$, and IADL $(\mathrm{p}=0.0046)$ scores.

No differences were seen between progressors and non-progressors in cognitive (MMSE) scores or behavioral symptoms, as assessed by NPI-12 total scores or subscores. When patients were categorized according to $\mathrm{AD}$ dementia severity, the progressor and non-progressor groups contained approximately the same proportions of those with mild, moderate, and moderately severe/severe disease. Most patients in both groups had received treatment for AD dementia. No difference was observed between groups in the tendency to fall.

Some significant differences in dependence levels between groups were apparent at baseline (all $\mathrm{p}<0.0001$ ). Progressors included more patients at lower dependence levels than non-progressors (e.g., $51.72 \%$ vs. $27.10 \%$ at dependence levels 0-2) and fewer at higher dependence levels ( $18.63 \%$ vs. $49.20 \%$ at levels $4-5$, respectively).

\section{Dependence Levels}

Dependence level distributions for the entire cohort at baseline and 18 months are shown in Figure 1. Compared with baseline, at 18 months fewer patients in the overall patient sample were categorized as level 2 (requiring the equivalent of limited or informal home care services) and, correspondingly, more patients were found to require care equivalent to that provided by assisted living plus nursing support or placement into a nursing home (levels 4 and 5). A small proportion of patients at both time points showed either no impairments (level 0) or little impairment (level 1).

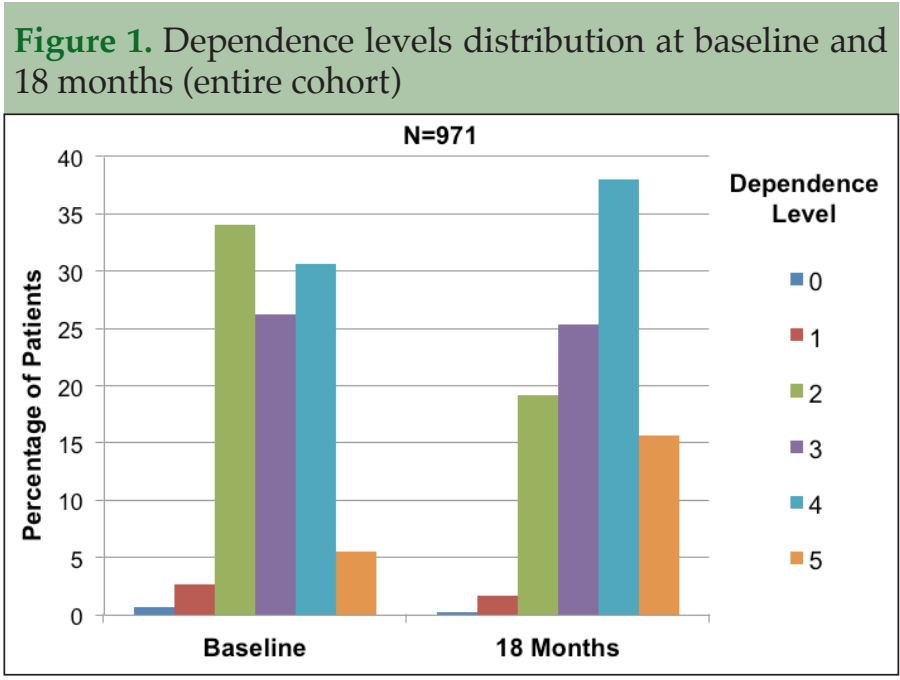

Patients with AD dementia (as determined by baseline MMSE cognitive scale) were assigned to one of six derived dependence levels, based on functional ability using the Alzheimer's Disease Cooperative Study Activities of Daily Living Inventory (ADCS-ADL), at baseline and 18 months. The dependence levels were: level 0 , no care needs; level 1 , independent living with check-ins; level 2, care equivalent to limited or informal home care services; level 3 , care equivalent to extensive home care with supervision or assisted living; level 4, care equivalent to that provided by assisted living plus nursing support; and level 5, care equivalent to a nursing home. Lower levels of dependence indicate better function.

Figure 2 illustrates the distribution of dependence levels according to AD dementia severity at baseline and at 18 months. The results demonstrate that both at baseline and after 18 months, patients with AD dementia at all levels of cognitive impairment had a range of dependence levels, although the distribution of patients at each dependence level differed according to severity group. 
Figure 2. Dependence level distributions according to AD dementia severity at baseline and 18 months

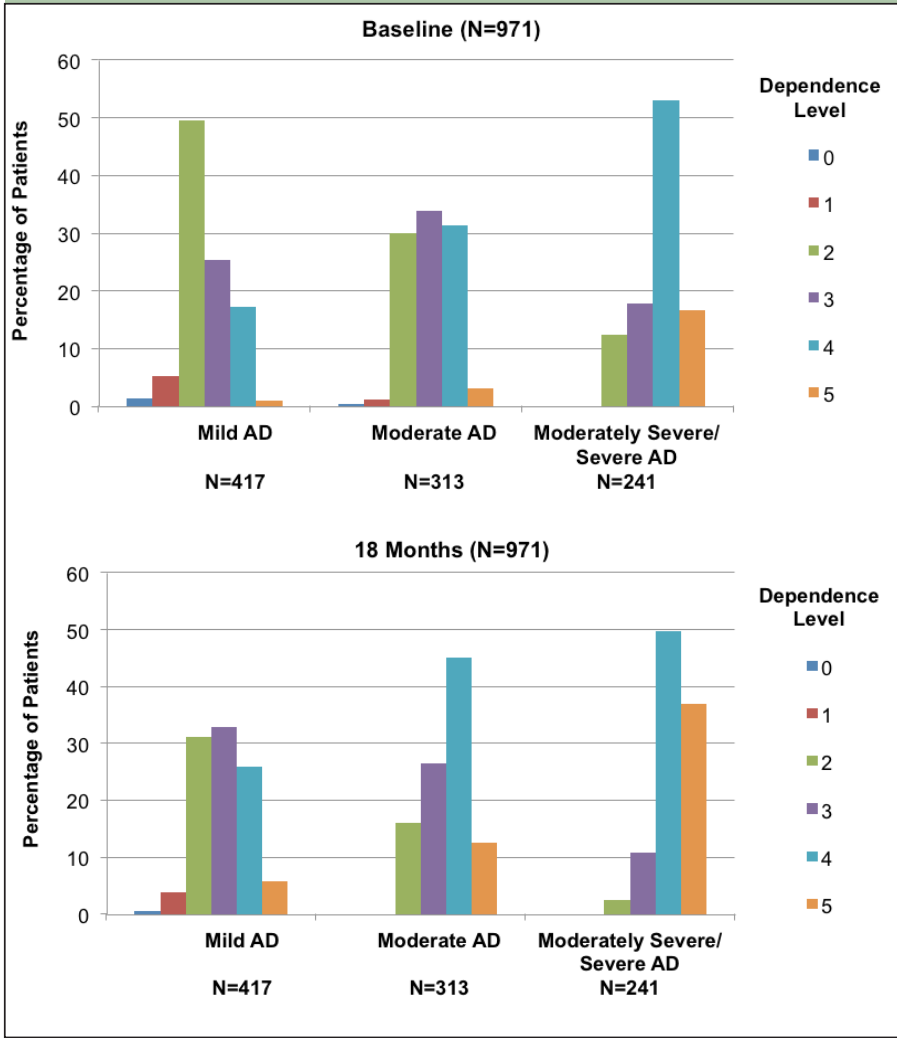

Patients were stratified according to disease severity by baseline cognitive function using the Mini-Mental State Examination (MMSE) as having mild (26-21), moderate $(20-15)$ or moderately severe/severe $(\leq 14)$ AD dementia. Patients with $\mathrm{AD}$ dementia were then assigned to one of six derived dependence levels, based on functional ability assessed using the Alzheimer's Disease Cooperative Study Activities of Daily Living Inventory (ADCS-ADL) at baseline and again at 18 months. The dependence levels were: level 0, no care needs; level 1, independent living with check-ins; level 2, care equivalent to limited or informal home care services; level 3, care equivalent to extensive home care with supervision or assisted living; level 4, care equivalent to that provided by assisted living plus nursing support; and level 5, care equivalent to a nursing home.

Note that no patients were determined to be at dependence level 0 or level 1 in the moderate $\mathrm{AD}$ group at 18 months or the moderately severe/severe group at baseline and at 18 months.

At baseline, $49.64 \%$ of those diagnosed with mild $\mathrm{AD}$ dementia required the equivalent of limited or informal home care services (dependence level 2), whereas $42.69 \%$ required at least the equivalent of extensive home care services with supervision or assisted living plus nursing support (levels 3-4). Less than 7\% (6.72\%) of patients with mild AD required no or little assistance (level 0 or 1 ) and $0.96 \%$ required the equivalent of nursing home care (level $5)$.

After 18 months, fewer patients $(31.18 \%)$ with mild AD dementia showed the equivalent of level 2 dependence compared with baseline, while $58.75 \%$ required higher levels of assistance (levels 3-4). Almost 6\% (5.76\%) required the equivalent of nursing home care.

In those with moderate $\mathrm{AD}$ dementia, the proportion of patients requiring the equivalent of assisted living plus nursing support or placement in a nursing home (levels 4 and 5) rose from $34.50 \%$ at baseline to $57.51 \%$ after 18 months. The majority of those with severe disease needed high levels of assistance at baseline $(69.71 \%)$, with a greater proportion requiring high levels of assistance at 18 months $(86.72 \%)$.

The pattern of relative progression versus nonprogression in dependence level observed in the overall cohort $(42.02 \%$ progression $/ 57.98 \%$ nonprogression) was similar in all groups of $\mathrm{AD}$ dementia severity. For those with mild AD dementia $(n=417)$, $39.57 \%$ progressed compared with baseline whereas $60.43 \%$ did not; corresponding values in the moderate AD dementia ( $\mathrm{n}=313)$ group were $44.73 \%$ and $55.27 \%$. Patients with severe AD dementia $(n=241)$ manifested high levels of dependence at baseline and this pattern became more prominent at 18 months, with $42.74 \%$ of patients becoming more functionally dependent and $57.26 \%$ not progressing during the 18-month follow-up. Of the 408 progressors, $294(72.06 \%)$ worsened by one level, $98(24.02 \%)$ worsened by two levels and $16(3.92 \%)$ worsened by more than two levels.

Figure 3. Association between baseline factors and dependence progression in patients with $\mathrm{AD}$ dementia

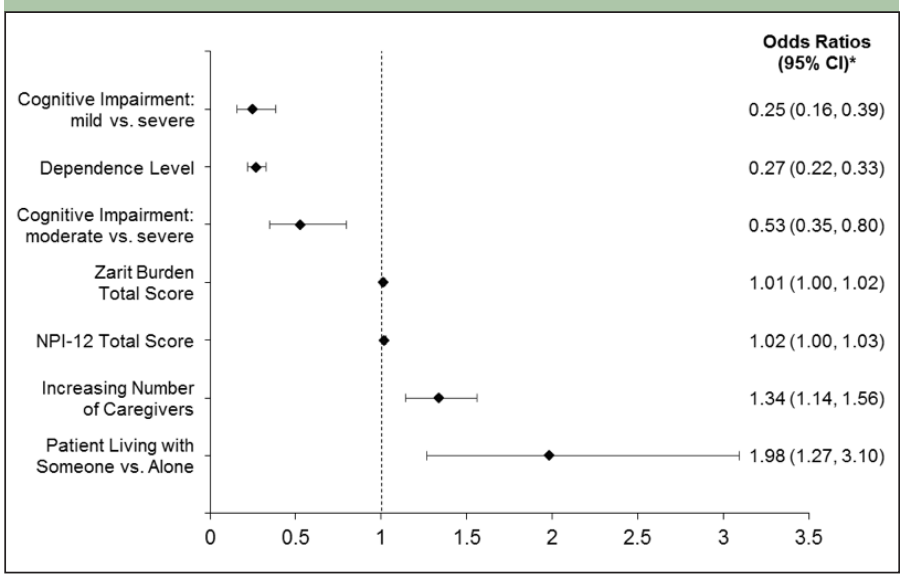

*Note that factors with $\mathrm{OR}<1$ indicate less likelihood of dependence progression, those with $\mathrm{OR}=1$ have no association with dependence progression, and those with $\mathrm{OR}>1$ are predictive of dependence progression.

\section{Relationship Between Dependence Levels and Other Outcome Measures}

In unadjusted correlations using Pearson correlation coefficients, a number of patient clinical outcome measures correlated significantly (all p-values <0.05) with levels of dependence at baseline and at 18 months. These included negative correlations for cognition (MMSE total score) ( $\mathrm{r}=-0.50$ at baseline, $\mathrm{r}=-0.57$ at 18 months) and patient QoL ( $\mathrm{r}=-0.37,-0.46$, respectively) and a positive correlation for patient neuropsychiatric disturbance $(\mathrm{r}=0.33,0.34$, respectively). From the caregiver's perspective, increased dependence correlated with greater caregiver time ( $\mathrm{r}=0.49,0.44$, respectively) and greater overall ZBI burden ( $\mathrm{r}=0.36,0.30$, respectively). Although there was no statistically significant correlation 


\begin{tabular}{|c|c|c|c|c|c|c|}
\hline Modeling & $\begin{array}{l}\text { Estimate for change } \\
\text { in dependence level }\end{array}$ & Standard error & \multicolumn{2}{|c|}{ Wald $95 \%$ confidence limits } & Wald Chi-square & Pr $>$ Chi-square \\
\hline \multicolumn{7}{|l|}{ Positive linear relationships } \\
\hline$\Delta \mathrm{ZBI}$ & 1.02 & 0.46 & 0.11 & 1.925 & 4.88 & 0.0272 \\
\hline$\Delta$ caregiver time (h/month) & 40.30 & 7.39 & 25.81 & 54.79 & 29.72 & $<0.0001$ \\
\hline$\triangle \mathrm{NPI}-12$ total score & 1.69 & 0.50 & 0.70 & 2.67 & 11.28 & 0.0008 \\
\hline$\Delta$ medical costs $(€ /$ month $)$ & 35.85 & 17.16 & 2.21 & 69.49 & 4.36 & 0.0367 \\
\hline$\Delta$ total societal costs $(€ /$ month $)$ & 299.57 & 55.68 & 190.44 & 408.70 & 28.95 & $<0.0001$ \\
\hline \multicolumn{7}{|l|}{ Negative linear relationships } \\
\hline$\triangle \mathrm{MMSE}$ & -1 & 0.17 & -1.33 & -0.67 & 36.03 & $<0.0001$ \\
\hline$\Delta \mathrm{EQ}-5 \mathrm{D}$ & -0.059 & 0.0098 & -0.08 & -0.0395 & 35.75 & $<0.0001$ \\
\hline
\end{tabular}

Abbreviations: AD, Alzheimer's Disease; ADL, activities of daily living; EQ-5D, EuroQol-5 Dimension; GLM, generalized linear model; MMSE, Mini-Mental State Examination; NPI, Neuropsychiatric Inventory; ZBI, Zarit Burden Interview. Note: $\Delta=$ change from baseline to 18 months. The changes in outcomes measures from baseline to 18 months were fitted using GLM models with normal distribution with identity link function. All models were adjusted for the core list of covariates. The model is listed as follows: change in outcome measure = baseline outcome measure + change in dependence level + the core list of covariates. The core list of covariates includes AD dementia severity, country, patient age, patient gender, number of comorbidities, baseline ADL total score, caregiver age, caregiver is spouse (yes/no), and caregiver works for pay (yes/no).

with patient direct medical costs at baseline ( $\mathrm{r}=0.05)$, dependence was positively correlated with these costs at 18 months $(\mathrm{r}=0.13)$, and total societal costs increased with greater dependence ( $\mathrm{r}=0.35,0.43$, respectively).

Table 2 shows the degree to which a change in one dependence level is strongly associated with changes in other clinical and outcome variables. Results of the GLM regression analyses controlling for a core list of covariates for each outcome suggest that positive linear relationships between change in dependence level and change in ZBI, caregiver time, NPI-12 total score, medical costs, and total societal costs, and negative linear relationships between change in dependence level and change in MMSE and EQ-5D are statistically significant.

\section{Factors Associated with Progressors versus Non-progressors}

Results of the logistic regression analysis shown in Figure 3 suggest that several patient baseline factors were significantly predictive of increasing dependence. They included greater cognitive impairment, such that patients with mild or moderate cognitive impairment were less likely than more severely impaired patients to have progressed a minimum of one dependency level over 18 months. However, having higher initial levels of dependence (e.g., level 2 vs. level 1) was associated with less risk of dependence progression over 18 months, as indicated by an odds ratio of 0.27 (95\% CI 0.22, 0.33). Other potential predictors of increasing dependency included the patient living with someone else compared to living alone, and having multiple caregivers. Age, education, gender, other comorbidities, neuropsychiatric symptoms, and elevated caregiver burden were not found to be predictive of dependence progression.

\section{Discussion}

Results of this large, observational cohort of patients with AD dementia at different stages of cognitive impairment reveal the heterogeneity of disease progression when it comes to functional abilities. After 18 months, even those with mild AD dementia required varied levels of support, with almost $60 \%$ needing the equivalent of extensive home care services with supervision/assisted living/nursing support and almost $6 \%$ the equivalent of nursing home care. As would be expected with AD disease progression, a general upward shift in dependence levels was observed over 18 months for all levels of AD dementia severity. However, individual variation was apparent and common. At all three levels of baseline AD dementia severity (mild, moderate and moderately severe/severe), over an 18 -month period between $55 \%$ and $60 \%$ of AD dementia patients manifested no change or an improvement in dependence while $40 \%$ to $45 \%$ became more dependent.

Identifying early in their disease those AD dementia patients who will manifest functional deficits and require assistance is critical for patients, family members, and healthcare systems. Having identified progressors and non-progressors in our cohort, we then went back to baseline data to examine possible early differentiating factors. Interestingly, no differences were seen between progressors and non-progressors in cognition (MMSE scores) or behavioral symptoms, tendency to fall, whether they were receiving treatment, or caregiver characteristics. With logistic regression we identified baseline factors associated with dependence progression, including greater cognitive impairment, having multiple caregivers, and living with another person. Conversely, having higher initial levels of dependence was associated with less risk of dependence progression. These seemingly contradictory findings may be explained by 
disease and/or measurement issues. The association between greater cognitive impairment at baseline and higher likelihood of increased dependence is consistent with prior research showing a pattern of cognitive decline preceding functional decline (25). The present analyses are also consistent with the expectation that higher dependence levels should be associated with other indicators of functional impairment, as evidenced by the finding that progressors were more likely to have multiple caregivers or live with another person. However, the categorical nature of dependence levels means that those at higher levels of dependence at baseline may not be as likely to progress due to ceiling effects.

A change of one dependence level can have practical consequences. For example, we estimate that a patient who declines one level in dependence requires 40 more hours of caregiving time per month, and incurs $€ 35.85$ per month more in medical costs and €299.57 per month more in total societal costs. Recently, Darba and Kaskens (9) used the DS to evaluate 343 patients with AD dementia in Spain. They found that with each additional 1 -point increase in DS, there was a $13.5 \%$ increase in direct medical care costs, a $25.3 \%$ increase in social care costs, and a $214.7 \%$ increase in informal care costs over 6 months. These data highlight the social and economic ramifications of deteriorating independence.

Selecting which outcome(s) to evaluate can be a challenge for an illness with as global an impact as $\mathrm{AD}$ dementia. Outcomes of interest reflect the needs and goals of patients, families, caregivers, clinicians, researchers, payers, and healthcare agencies. While cognition is traditionally thought to be the chief variable of interest in $\mathrm{AD}$ dementia, and is the hallmark impairment of the disease (26), assessment of functional abilities, behavior, caregiver burden, QoL, resource utilization, and costs also provide worthwhile information, which can be used to stage AD dementia and assess disease impact (27). Once a relevant outcome(s) of interest is chosen, it is then important to select an instrument that will provide valid and meaningful data $(27,28)$.

Our results support the suggestions of Zhu et al. (6, 29) that dependence is a distinct component of disability in $\mathrm{AD}$ dementia, and that assessment of patient function and dependence provides information not available from the MMSE or other cognitive measures. Further, increases in dependence are associated with other indicators of increasing disease severity such as more caregiver time, higher caregiver burden, and higher care costs. The additional association between cognition and dependence suggests that dependence is a useful summary measure of AD severity.

We believe that dependence levels may be considered as interim clinical milestones that reflect AD dementiarelated functional deficits and can be a useful metric to monitor disease course. Measuring dependence levels may offer valuable information for tracking, managing, and treating patients with AD dementia. These assessments may be particularly informative when characterizing such patients in the mildest stage of disease, a time when discrete clinical milestones are difficult to ascertain. Whether measurable shifts in dependency can occur within 18 months with enough sensitivity to make it an appropriate 18-month endpoint for phase 3 clinical trials remains to be determined, but this metric may prove to be useful for longer-term trials, trials enriched with a population more likely to progress, or observational studies.

\section{Strengths of the Study}

The GERAS study included a large population of patients representative of the $\mathrm{AD}$ dementia continuum. The aim of the GERAS study was to address some of the limitations of previous cost studies by using wellestablished, standardized methods for assessing resource use and caregiver time over a longer follow-up period. The study also provides unique information on the societal costs of AD dementia in community-dwelling patients, both across different severity levels and between countries (14).

\section{Study Limitations}

One limitation of GERAS is that the study sample did not include the entire spectrum of functional impairment. Specifically, enrolled patients were required to be community dwelling, thus excluding those who were severely impaired. In addition, patients with cognitive impairment who did not yet show signs of dementia (prodromal AD) were also excluded. This limits the external validity of the results. The frequency of users of $\mathrm{AD}$ medications may be overestimated because study centers were mostly specialist clinics (14).

Another limitation is that although about $40 \%$ of the patients in the moderately severe/severe group had an MMSE score of $<10(14)$, the cut-offs used in the GERAS study may bias the moderately severe/severe AD dementia group toward those with less-severe symptoms. Additionally, because observed discontinuation rates tend, in general, to be higher in those with greater cognitive and functional disabilities, dependence progression may have been underestimated in our study, which focused only on completers. Also, differences in patient characteristics between AD dementia severity groups in each country may have confounding effects on resource utilization and associated costs, but these have not been taken into account. Lastly, cultural differences between countries regarding the care of patients with $\mathrm{AD}$ dementia, as well as differences in healthcare systems, mean that the resource utilization and cost data from the three countries in the GERAS study (France, Germany, 
and the UK) may limit the generalizability of the findings (14). It should also be noted that patients were enrolled in GERAS based on NINCDS-ADRDA clinical criteria; however, no biomarker information or genotype status was captured. Further research is warranted to evaluate the power of these factors to predict progression status.

\section{Conclusions}

Understanding the types of changes that patients with $\mathrm{AD}$ dementia undergo can help determine what sorts of milestones may be suitable for clinical use and/or use in clinical trials and observational studies. Our study demonstrated that dependence levels may provide unique information on clinical progression beyond what is captured with a cognitive measure. We have also shown the pragmatic consequences of increasing dependence on patients, caregivers, and costs. Identification of several baseline factors associated with functional deterioration opens up possibilities for early intervention.

\section{Funding: This study was supported by Eli Lilly and Company.}

Acknowledgments: The authors would like to acknowledge Amy Rothman Schonfeld, Gill Gummer and Caroline Spencer (Rx Communications, Mold, UK) for assistance with the preparation of this article, funded by Eli Lilly and Company. The authors would also like to thank Dr. Yaakov Stern for sharing his insights and expertise during the course of this research.

Disclosures: KK-W, JSA, MB and WY are all employees and minor shareholders of Eli Lilly and Company. SG has received clinical trial support from Eli Lilly and Company, Roche, TauRx and Lundbeck Pharmaceuticals, was a DSMB member for ADCS, ATRI, API and Eisai, was a scientific advisor for AbbVie, Advantage, Alzheon, Axovant, Boehringer-Ingelheim, Firalis, Heptares, IntelGen, Kalgene, Eli Lilly and Company, Lundbeck Pharmaceuticals, Novartis, Otsuka, Servier, Sanofi, Schwabe, Takeda, TauRx, TVM Capital and Roche. DMR has served as a consultant for Eli Lilly and Company, Biogen Idec, Lundbeck Pharmaceuticals, and serves as a member of the Scientific Advisory Board for Neurotrack. DG has received personal fees from Biomed Central, vTv Therapeutics, Janssen Immunotherapy, Prothena Corporation, the Michael J Fox Foundation, and has received grants from California Institute for Regenerative Medicine and the Michael J Fox Foundation.

\section{References}

1. McKhann G, Knopman DS, Chertkow H, et al. The diagnosis of dementia due to Alzheimer's disease: Recommendations from the National Institute on Aging-Alzheimer's Association workgroups on diagnostic guidelines for Alzheimer's disease. Alzheimers Dement 2011;7:263-269.

2. Mitchell AJ, Meader N, Pentzek M. Clinical recognition of dementia and cognitive impairment in primary care: a meta-analysis of physician accuracy. Acta Psychiatr Scand 2011;124:165-183.

3. Stella F, Forlenza OV, Laks J, et al. Caregiver report versus clinician impression: disagreements in rating neuropsychiatric symptoms in Alzheimer's disease patients. Int J Geriatr Psychiatry 2015 9;30:1230-1237.

4. Kahle-Wrobleski K, Coley N, Lepage B, et al., for the PLASA/DSA Group. Understanding the complexities of functional ability in Alzheimer's disease: more than just basic and instrumental factors. Curr Alzheimers Res 2014;11:357-366.

5. Molnar FJ, Man-Son-Hing M, Fergusson D. Systematic review of measures of clinical significance employed in randomized controlled trials of drugs for dementia. J Am Geriatr Soc 2009;57:536-546.

6. Zhu CW, Leibman C, Townsend R, et al. Bridging from clinical endpoints to estimates of treatment value for external decision makers. J Nutr Health Aging 2009;13:256-259.
7. Scherer RK, Scarmeas N, Brandt J, et al. The relation of patient dependence to home health aide use in Alzheimer's disease. J Gerontol A Biol Sci Med Sci 2008;63:1005-1009.

8. Jones RW, Romeo R, Trigg R, et al., for the DADE Investigator Group. Dependence in Alzheimer's disease and service use costs, quality of life, and caregiver burden: The DADE study. Alzheimers Dement 2015 11:280-290. DOI: $10.1016 /$ j.jalz.2014.03.001.

9. Darba J, Kaskens L. Relationship between patient dependence and direct medical- social-, indirect - and informal-care costs in Spain. ClinicoEcon Outcomes Res 2015;7:387-395.

10. Gauthier S, Leuzy A, Racine E, et al. Diagnosis and management of Alzheimer's disease: past, present and future ethical issues. Prog Neurobiol 2013;110:102-113.

11. Lenderking MR, Wyrwich KW, Stolar M, et al. Reliability, validity, and interpretation of the dependence scale in mild to moderately severe Alzheimer's disease. Am J Azheimers Dis Other Dement 2013; 28:738-749.

12. Brickman AM, Riba A, Bell K, et al. Longitudinal assessment of patient dependence in Alzheimer disease. Arch Neurol 2002;59:1304-1308.

13. Stern Y, Albert SM, Sano M, et al. Assessing patient dependence in Alzheimer's disease. J Gerontol 1994;49:M216-222.

14. Wimo A, Reed CC, Dodel R, et al. The GERAS study: a prospective observational study of costs and resource use in community dwellers with Alzheimer's disease in three European countries - study design and baseline findings. J Alzheimers Dis 2013;36:385-399.

15. Kahle-Wrobleski K, Andrews JS, Belger M, et al. Clinical and economic characteristics of milestones along the continuum of Alzheimer's disease: Transforming functional scores into levels of dependence. J Prev Alzheimers Dis Feb 2015;2:115-120.

16. McKhann G, Drachman D, Folstein M, et al. Clinical diagnosis of Alzheimer's disease: report of the NINCDS-ADRDA Work Group under the auspices of Department of Health and Human Services Task Force on Alzheimer's Disease. Neurology 1984;34:939-944.

17. Folstein MF, Folstein SE, McHugh PR. Mini-mental state. A practical method for grading the cognitive state of patients for the clinician. J Psychiatr Res 1975;12:189-198.

18. National Institute of Health and Clinical Excellence. NICE technology appraisal guideline 217. Donepezil, galantamine, rivastigmine and memantine for the treatment of Alzheimer's disease. http:/ / www.nice.org. uk/guidance/TA217. March 2011. Accessed March 19, 2015.

19. Galasko D, Bennett D, Sano M, et al. An inventory to assess activities of daily living for clinical trials in Alzheimer's disease. The Alzheimer's Disease Cooperative Study. Alzheimer Dis Assoc Disord 1997;11(Suppl 2):S33-S39.

20. The EuroQol Group. Euro-Qol - a new facility for the measurement of healthrelated quality of life. Health Policy 1990;16:199-208.

21. Cummings JL, Mega M, Gray K, et al. The Neuropsychiatric Inventory: assessing psychopathology in dementia patients. Neurology 1997;48(Suppl 6):S10-S16.

22. Wimo A, Wetterholm AL, Mastey V, et al. Evaluation of resource utilization and caregiver time in anti-dementia drug trials: a quantitative battery. In Wimo A, Jonsson B, Karlsson G, (eds). The Health Economics of Dementia, 1998. John Wiley and Sons, London: pp. 465-499.

23. Zarit SH, Reever KE, Bach-Peterson J. Relatives of the impaired elderly: correlates of feelings of burden. Gerontologist 1980;20:649-655.

24. Dodel R, Belger M, Reed C, et al. Determinants of societal costs in Alzheimer's disease: GERAS study baseline results. Alzheimer's Dement 2015:11:933-945.

25. Liu-Seifert H, Siemers E, Sundell K, et al. Cognitive and functional decline and their relationship in patients with mild Alzheimer's dementia. J Alzheimers Dis 2015;43:949-955. doi: 10.3233/JAD-140792.

26. Vellas B, Carrillo MC, Sampaio C, et al, EU/US/CTAD Task Force Members. Designing drug trials for Alzheimer's disease: what we have learned from the release of the phase III antibody trials: a report from the EU/US/CTAD Task Force. Alzheimer's Dement 2013:9:438-444.

27. Green Park Collaborative. Evidence Guidance Document. Design of Clinical Studies of Pharmacologic Therapies for Alzheimer's Disease. 11/04/13. http: / / www.cmtpnet.org/docs/resources / GPC_Evidence_Guidance_ Document_-_Alzheimers_Disease.pdf

28. Doody RS, Thomas RG, Farlow M, et al., for the Alzheimer's Disease Cooperative Study Steering Committee; and Siemers E, Liu-Seifert H, and Mohs for the Solanezumab Study Group. Phase 3 trials of solanezumab for mild-to-moderate Alzheimer's disease. N Engl J Med 2014;370:311-321.

29. Zhu CW, Leibman C, McLaughlin T, et al. The effects of patient function and dependence on costs of care in Alzheimer's Disease. J Am Geriatr Soc 2008;56:1147-1503. 